\title{
Immunological profiles of beekeepers and patients allergic to bee venom: understanding tolerance to bee stings
}

\author{
Berta Ruiz ${ }^{1}$, Ana Navas ${ }^{2}$, Pilar Serrano ${ }^{2}$, Ipek Guler ${ }^{2}$, Maria Espinazo Romeu ${ }^{2}$, Corona \\ Alonso $^{2}$, Aurora Jurado ${ }^{3}$, and Carmen Moreno-Aguilar ${ }^{2}$ \\ ${ }^{1}$ Maimonides Biomedical Research Institute of Cordoba (IMIBIC)/ Reina Sofia University \\ Hospital/ University of Cordoba \\ ${ }^{2}$ Maimonides Biomedical Research Institute of Cordoba (IMIBIC)/ Reina Sofia University \\ Hospital/ University of Cordoba \\ ${ }^{3}$ Maimonides Biomedical Research Institute of Cordoba (IMIBIC)/ Reina Sofia University \\ Hospital
}

June 17, 2020

\begin{abstract}
Background. Although exposure to stings has been identified as the main risk factor for anaphylaxis due to Hymenoptera venom allergy, professional beekeepers receive hundreds of them yearly without developing systemic reactions. This study aims to analyse the immunological profile of people exposed to bee stings. Methods. A cross-sectional study was conducted. Participants were recruited and classified into three groups: allergic patients (AP) experiencing systemic reactions after bee stings, with a positive intradermal test and specific IgE (sIgE) to Apis mellifera venom (AmV); tolerant beekeepers (TBK) receiving [?] 50 stings/year; and healthy non-exposed controls (HC). Serum levels of sIgE and specific IgG4 (sIgG4) to AmV, rApi m1, rApi m2, rApi m3, Api m4, rApi m5 and rApi m10, as well as AmV-induced basophil degranulation, percentage of T-cell subsets, Treg cells and IL-10 production were measured. Results. APs had high levels of sIgE to AmV and all allergic components $(\mathrm{p}<0.001)$ together with a high basophil activation rate $(\mathrm{p}<0.001)$ compared to TBKs. Conversely, compared to TBKs, APs showed lower levels of sIgG4 $(\mathrm{p}<0.001)$ and IL-10 $(\mathrm{p}<0.001)$ as well as a reduced CTLA-4+ Treg population $(\mathrm{p}=0.001)$, together with enhanced Helios + Treg $(\mathrm{p}<0.004)$, Th1 $(\mathrm{p}=0.008)$, Th2 $(\mathrm{p}=0.004)$ and Th17 ( $\mathrm{p}=0.007)$ populations. Conclusion. Two different profiles were identified: the allergic profile is well defined by parameters of IgE response, some of them also present in the tolerant beekeeper profile, which is strongly marked by Treg activity.
\end{abstract}

\section{Hosted file}

Manuscript B. Ruiz-20200615 with fig.doc available at https://authorea.com/users/334258/ articles/460280-immunological-profiles-of-beekeepers-and-patients-allergic-to-bee-venomunderstanding-tolerance-to-bee-stings

\section{Hosted file}

Graphical abstract 20200615.pptx available at https://authorea.com/users/334258/articles/ 460280-immunological-profiles-of-beekeepers-and-patients-allergic-to-bee-venomunderstanding-tolerance-to-bee-stings 